\title{
A $\lambda$-CUT APPROXIMATE ALGORITHM FOR GOAL-BASED BILEVEL RISK MANAGEMENT SYSTEMS
}

\author{
YA GAO*. GUANGQUAN ZHANG and JIE $\mathrm{LU}^{\ddagger}$ \\ Faculty of Information Technology, Unitersity of Technology \\ Sydney. PO Box 123, Broaduay, NSW 2007, Australiu \\ *yagao@it.uts, edurau \\ tzhangg@il.uts. edu.ou \\ †jeluôt uts edu an \\ THARAM DILLON \\ Digital Eeosystems and Business Intelligence Institute \\ Curtin University of Technology, Perth, WA 685, Australio \\ tharam, dillongebs, curtin, edu, an \\ XIANYI ZENG

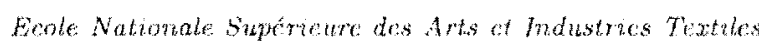 \\ 9 rue de lWmatage 59100 Roubaix, France \\ rumbengonsantofy
}

\begin{abstract}
Bilevel programming techiques are developed for decentralized decision problems with decsion makers located in two levels. Both upper and lower decision makers, termed as leader and follower, try to optimize their own objectives in solution procedure but are affected by those of the other lavels. When a bilevel decision model is built with fuzzy cocticients and the learder and/or lollower have goals for their objectives, wo call it fuzzy goal bilevel (FGBL) decision problem. This paper fitst proposes a $\lambda$-cut set based FCBL modal. A programmable $\lambda$-cut approximate algorithn is then presented in letail. Based on this algorithm, a FGBS software system is developed to reach solutions for FGBI, decision problems. Finally, two examples are given to illustrate the application of the proposed algorithm.
\end{abstract}

Keywords: Bilevel decision making; goal programming; fuzcy setw; optimization; risk management.

\section{Introduction}

Bilevel programming techniques, initiated by Von Stackelberg, ${ }^{25}$ are mainly developed for solving decentralized managenent problems with decision makers in a two-level hierarchy. The upper decision maker is termed leader ant the lower the follower. ${ }^{2}$ Fuzzy bilevel programming techniques, which handle bilevel decision

¥Corresponding author. 
problems when coefficients are described by fuzzy sets, ${ }^{30}$ are recognized effective on analyzing potential risks and generating warnings in risk management.

The investigation of bilevel decision problens is strongly motivated by real workl applications, and bilevel programming techniques have been applied with remarkable success in different donains such as decentralized resource planning. ${ }^{28}$ electronic power market, ${ }^{9}$ logistics. ${ }^{29}$ civil engineering. ${ }^{3}$ and road network managenent. ${ }^{6.7}$ For risk management, which ainss to measure and assess any risk and develop strategies to manage it, ${ }^{26}$ bilevel programming techniques play significant roles as well. Decision makers face the challenge of allocating supply resources, transportation ability, rescue aid and whatever to minimize the effect of threat. These decision makers may be located at different levels within a management network and thus have inconsistent concerns. For example, when a severe earthquake occurs, ${ }^{6}$ the roadway systems usually get different degrees of damage. which reduces the through capacity and causes traffic congestion. The commander of an Emergency-Response Center, in the upper level, aims at allowing traffic to go through the disaster areas as much as possible within the roadway capacitv, while the road users, located at the lower level. always choose the shortest route to actualize emergency rescues. The decision from the commander and the road users will inevitably influence the choice from each other. In this situation. bilevel programming should be a suitable technigue to solve this decision problem.

Fuzzy numbers, which are used for representing mumerical quantities in a vague environment. ${ }^{27}$ have been applied in subsequent research on bilevel decision problems. Shih et al. ${ }^{24}$ and $\mathrm{Lai}^{13}$ first applied fuzzy set approach to bilevel decision problems. Their method. however, sometines might canse a final undesirable solution due to the inconsistency between fuzzy goals of the objective functions and the decision variables. ${ }^{22}$ To overcone this problem, Salawa et al. ${ }^{22}$ developed an interactive fuzzy set approach by deriving a satisfactory solution and updating the satisfactory degrees of decision makers with considerations of overall satisfactory balance among all levels. In our research lab, an approximation approach has been developed ${ }^{8,30}$ based on framework building and models formatting. ${ }^{16,17}$ Solutions can be reached by solving associated multiple ohjectives bilevel decision problem under different cut sets.

Goal programming was originally proposed by Charnes and Cooper ${ }^{4}$ in 1961 for a linear model. It has been further developed by Lee, ${ }^{14}$ Ignizio, ${ }^{11.12}$ Charnes and Cooper. ${ }^{5}$ Recent research on goal programming can be found fron Refs. 10. 15, 18 20. Goal programming requests a decision maker to set a goal for the objective that he/she wishes to attain. A preferred solution is then defined to minimize the deviation from the goal. Therefore. goal programming seems to yield a satisfactory solution rather than an optimal one. In fuzzy bilevel decision problems. when both a leader and follower set goals for their objectives respectively, the problen becones a FGBL decision problem. which is addressed by this study.

This paper is organized as follows. After the introduction, Sec. 2 reviews related definitions and theorems of FGBL programming. In Sec. 3 , a $\lambda$-cut set based FGBL 
model and a $\lambda$-cut approximate algorithin to solve FGBL problems are presented. Meanwhile, a FGBL software system which inplemented the proposed algorithn is described. A numerical example and a case-based exaniple on traffic management in a disaster area are shown in Sec. 4. Conclusions and further study are discussed in Sec. 5.

\section{Preliminaries}

In this section, some defintions and formulations used in subsequent sections are presented.

Throughont this paper, $\mathbb{R}$ represents the set of all real numbers, $\mathbb{R}^{n}$ is $n$ dimensional Euclidean space, $F^{*}(\mathbb{R})$ and $F^{*}\left(\mathbb{R}^{n}\right)$ are the set of all finite fuzzy numbers and the set of all $n$-dimensional finite fuzzy numbers on $\mathbb{R}^{\prime \prime}$. respectively.

Definition 2.1. (Ref. 21) The $\lambda$-cut set of a fuzzy set $\tilde{A}$ is defined as an ordinary set $A_{\lambda}$ for which the degree of its membership function exceeds the level $\lambda$ :

$$
A_{\lambda}=\left\{x \mid \mu_{A}(x) \geq \lambda\right\}, \quad \lambda \in[0,1] .
$$

$A_{\lambda}$ is a nonempty bounded closed interval and it can be denoted by

$$
A_{\lambda}=\left[A_{\lambda}^{L} \cdot A_{\lambda}^{R}\right]
$$

where $A_{\lambda}^{L}$ and $A_{\lambda}^{F}$ are the lower and upper bounds of the interval, respectively.

Definition 2.2. (Ref. 30) For any n-dimensional fuzzy numbers $\tilde{a} . \tilde{b} \in F\left(\mathbb{R}^{\prime \prime}\right)$. under a certain satisfactory degree $\alpha$, we define

$$
\tilde{a} \preceq_{a} \tilde{b} \text { iff } a_{i \lambda}^{L} \leq b_{i \lambda}^{L} \text { and } a_{i \lambda}^{R} \leq b_{i \lambda}^{R}, \quad i=1,2, \ldots, n . \quad \forall \lambda \in[\alpha, 1],
$$

where $\alpha$ is the adjustable satisfactory legres, which means, when comparing two fuzzy numbers all values with membership grades smaller than $\alpha$ are neglected.

Definition 2.3. A fuzzy linear bilevel (FLBL) decision problem is defined a $3^{3 n}$ For $x \in X \subset \mathbb{R}^{n}, y \in Y \subset \mathbb{R}^{m}, F: X \times Y \rightarrow F^{*}(\mathbb{R})$, and $f: X \times Y \rightarrow F^{*}(\mathbb{R})$.

$$
\begin{aligned}
& \min _{x \in X} F(x, y)=\tilde{c}_{1} x+\tilde{d}_{1} y \\
& \text { subject to } \tilde{A}_{1} x+\tilde{B}_{1} y \preceq_{\alpha} \tilde{b}_{1} \\
& \min _{y \in Y^{\prime}} f(x, y)=\tilde{c}_{2} x+\tilde{d}_{2} y \\
& \text { subject to } \tilde{A}_{2, x}+\tilde{B}_{2} y \preceq_{\alpha} \tilde{b}_{2},
\end{aligned}
$$

where $\tilde{c}_{1}, \tilde{c}_{2} \in F^{*}\left(\mathbb{R}^{n}\right), \tilde{d}_{1}, \tilde{d}_{2} \in F^{*}\left(\mathbb{R}^{m}\right), \tilde{b}_{1} \in F^{*}\left(\mathbb{R}^{p}\right), \tilde{b}_{2} \in F^{* *}\left(\mathbb{R}^{q}\right), \tilde{A}_{1}=\left(\bar{a}_{i j}\right)_{p \times n}$. $\tilde{a}_{i j} \in F^{*}(\mathbb{R}) . \tilde{B}_{1}=\left(\tilde{b}_{i j}\right)_{p^{\prime} \times m}, \tilde{b}_{i, j} \in F^{*}(\mathbb{R}), \tilde{A}_{2}=\left(\tilde{e}_{i, i}\right)_{q \times n}, \tilde{e}_{i j} \in F^{*}(\mathbb{R}), \tilde{B}_{2}=$ $\left(\tilde{s}_{i j}\right)_{q \times m}, \tilde{s}_{i j} \in F^{*}(\mathbb{R})$, and $F^{*}(\mathbb{R})$ is the set of all finite fuzzy numbers. 
Theorem 2.1. (Ref. 30) For $x \in X \subset \mathbb{R}^{\prime \prime}, y \in Y \subset \mathbb{R}^{m}$, if all the fuzzy coefficients $\tilde{a}_{i j}, \tilde{b}_{i j}, \tilde{e}_{i j}, \tilde{s}_{i j}, \tilde{b}_{i}, \tilde{c}_{i}$, and $\tilde{d}_{i}$ have membership functions in FLBL problem (1):

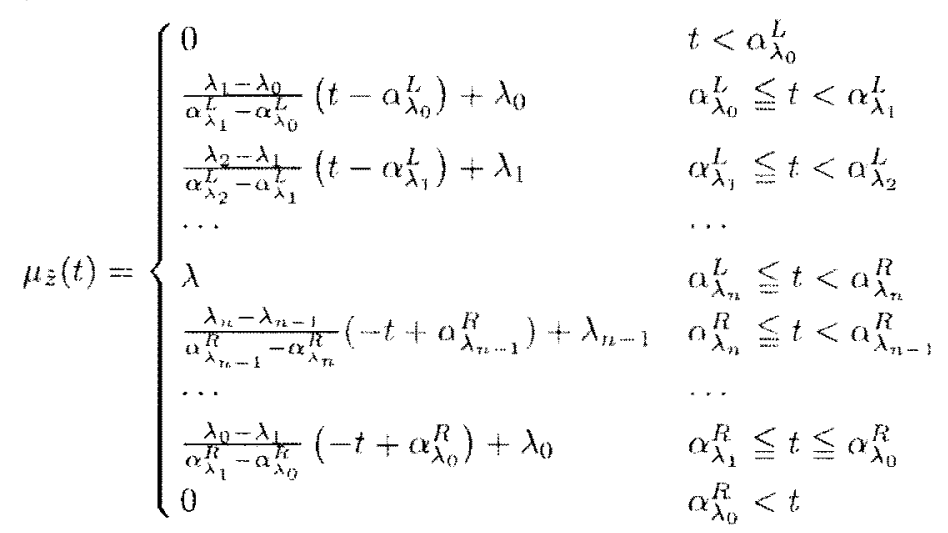

where $\tilde{z}$ denotes $\tilde{a}_{i j}, \tilde{b}_{i j}, \tilde{\epsilon}_{i j}, \tilde{s}_{i j}, \tilde{b}_{i}, \tilde{c}_{i}$, and $\tilde{d}_{i}$, respectively; then, it is the solution of problem (1) that $\left(x^{*} \cdot y^{*}\right) \in \mathbb{R}^{n} \times \mathbb{R}^{m}$ satisfining

$$
\begin{aligned}
& \min _{x \in X}(F(x, y))_{\lambda_{0}}^{L_{L}}=c_{1}{ }_{\lambda_{0}}^{L} x+d_{1}{ }_{\lambda_{0}}^{L} y,
\end{aligned}
$$

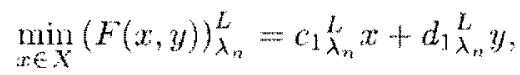

$$
\begin{aligned}
& \left.\min _{x \in X}(F(x, y))_{\lambda_{0}}^{R}=c_{1}\right]_{\lambda_{0}}^{R} x+d_{1} d_{\lambda_{0}}^{R} y . \\
& \min _{x \in X}(F(x, y))_{\lambda_{n}}^{R}=\mathrm{e}_{\lambda_{\lambda_{n}}}^{R} x+d_{1}{ }_{\lambda_{n}}^{R} y, \\
& \text { subject to } A_{1} \lambda_{\lambda_{0}}^{L} x+B_{1}{ }_{\lambda_{1}}^{L} y \leqq b_{1}{ }_{\lambda_{1}}^{L} \text {, }
\end{aligned}
$$

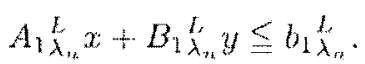

$$
\begin{aligned}
& A_{i}{ }_{\lambda_{1}}^{F} x+B_{1}^{R} y \leq b_{1}^{R} \lambda_{\lambda_{0}}^{R} \text {, } \\
& A_{1} \stackrel{R}{R} x+B_{\lambda_{n}}^{R} y \leqq b_{\lambda_{n}}^{R}, \stackrel{R}{R}, \\
& \min _{y \in Y}(f(x, y))_{\lambda_{0}}^{L_{1}}=c_{\lambda_{\lambda_{0}}}^{L} x+d_{2}{ }_{\lambda_{0}}^{L} y, \\
& \min _{y \in Y^{\prime}}(f(x, y))_{\lambda_{n t}}^{L}=c_{2} \dot{\lambda}_{\lambda^{2}} x+d_{2} \underset{\lambda_{n}}{L} y, \\
& \min _{y \in Y^{Y}}(f(x, y))_{\lambda_{0}}^{R}=c_{2}^{R} x+d_{2 \lambda_{0}}^{R} y \\
& \min _{y \in Y^{\prime}}(f(x, y))_{\lambda_{n}}^{R}=c_{2}^{R} x+d_{\lambda_{n}}^{R} y
\end{aligned}
$$




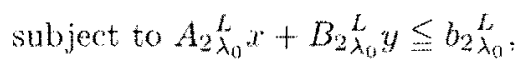

$$
\begin{aligned}
& A_{2 \lambda_{n}}^{L} x+B_{2}{ }_{\lambda_{n}}^{L} y \leqq b_{2 \lambda_{n i}}^{L}, \\
& A_{2 \lambda_{0}}^{R} x+B_{2 \lambda_{0}}^{R} y \leqq b_{2}^{R}, \\
& A_{2 \lambda_{n 2}}^{R} x+B_{2 \lambda_{n}}^{R} y \leq b_{2 \lambda_{n i}}^{R}
\end{aligned}
$$

\section{A $\lambda$-Cut Approximate Algorithm for Fuzzy Goal Bilevel Decision Problems}

First, we give the definition of a multiple objective bilevel (MOBL) decision problem:

Definition 3.1. For $x \in X \subset \mathbb{R}^{\prime \prime}, y \in Y \subset \mathbb{R}^{n}$, a MOBL decision problem is defined as

$$
\begin{aligned}
& \min _{x \in X} F(x, y) \\
& \text { subject to } G(x, y) \leq 0, \\
& \min _{y \in Y^{\prime}} f(x, y) \\
& \text { subject, to } g(x, y) \leq 0,
\end{aligned}
$$

where $F: \mathbb{R}^{n} \times \mathbb{R}^{m} \rightarrow \mathbb{R}^{k}, G: \mathbb{R}^{n} \times \mathbb{R}^{m} \rightarrow \mathbb{R}^{n}, f: \mathbb{R}^{n} \times \mathbb{R}^{m} \rightarrow \mathbb{R}$, and $y: \mathbb{R}^{n} \times \mathbb{R}^{n} \rightarrow \mathbb{R}^{\prime}$.

Associated with MOBL problem (1). some definitions are listed below:

\section{Definition 3.2.}

(1) Constraint region of MOBL problem (4)

$$
S \triangleq\{(x, y): x \in X, y \in Y, G(x, y) \leq 0, g(x, y) \leq 0\} .
$$

It refers to all possible combinations of choices that the leader and follower may nake.

(2) Projection of $S$ onto the leader's decision space

$$
S(X) \triangleq\{x \in X: \exists y \in Y, G(x, y) \leq 0 . g(x, y) \leq 0\} .
$$

(3) Feasible set for the follower $\forall x \in S(X)$ :

$$
S(x) \triangleq\left\{y \in Y^{*}:(x, y) \in S\right\} .
$$

(4) Follower's rational reaction set for $x \in S(x)$ :

$$
P(x) \triangleq\{y \in Y: y \in \operatorname{argmin}[f(x, \hat{y}): \dot{y} \in S(x)]\} .
$$


where $\operatorname{argmin}[f(x, y): \dot{y} \in S(x)]=\{y \in S(x): f(x, y) \leq f(x, y), y \in S(x)\}$. which means, the follower observes the leader's action and reacts by selecting $y$ from his/her feasible set to minimize his/her objective function.

(5) Inducible region

$$
I R \triangleq\{(x, y):(x, y) \in S, y \in P(x)\}
$$

which represents the set over which the leader mav optimize his or her objective.

To ensure that (4) is well posed, it is assumed that $S$ is nonempty and compact, and that for all decisions taken by a leader, the follower has some room to respond: i.e. $P(x) \neq \emptyset$. Thus, in terms of the above notation, a MOBL problem can be written as

$$
\min \{F(x, y):(x, y) \in I R\}
$$

Goals given for objectives of a leader and follower in (1) are denoted by fuzzy numbers $\tilde{g}_{L}$ and $\tilde{g}_{F}$ with memlership functions $\mu_{g_{1}}$ and $\mu_{y_{F}}$, respectively. and our concern is to make the objectives of both the leader and the follower as near to their goals as possible. The differences between $F(x, y)$ and $\tilde{g}_{L}, f(x, y)$ and $\tilde{g}_{F}$ are usually defined as deviation functions. Initiated by the idea of Theorem 2.1, we use $\lambda$-cut set of fuzzy nunber to format a FGBL morlel as in Definition 3.3.

Definition 3.3. The $\lambda$-cut set based FGBL model is defined as

$$
\begin{aligned}
& \min _{x \in X}\left|c_{1 \lambda_{j}}^{L} x+d_{1, \lambda,}^{L} y-g_{L, \lambda_{j}}^{L}\right| \text {. } \\
& \min _{x \in X^{\prime}}\left|c_{1 \lambda_{j}}^{R} x+d_{1 \lambda_{j}}^{R} y-g_{L, \lambda_{j}}^{R}\right| \text {, } \\
& \text { subject to } A_{1 \lambda_{j}}^{L} x_{i}+B_{1 \lambda_{j}}^{L} y \leqq b_{1}{ }_{\lambda_{i}}^{L} \text {, } \\
& A_{1 \lambda_{j}}^{R} x+B_{1 \lambda_{j}}^{R} y \leqq b_{1 \lambda_{j}}^{R} \\
& \min _{y \in Y}\left|c_{2 \lambda_{j}}^{L} x+d_{2 \lambda_{j}}^{L} y-q_{j \lambda_{j}}^{L}\right| . \\
& \min _{y \in \mathrm{Y}^{\prime}} \mid c_{2 \lambda}, x+d_{2,}^{R}, y-g g_{\lambda,}^{R}, \\
& \text { subject to } A_{2 \lambda_{1}}^{L} x+B_{2}{ }_{\lambda_{3}}^{L} y \leqq b_{2} \lambda_{\lambda} \text {, } \\
& A_{2 \lambda_{j}}^{R} x+B_{2 \lambda_{j}}^{R} y \leqq b_{2 \lambda_{j}}^{R} \text {. } \\
& j=0,1,2, \ldots, l \text {. }
\end{aligned}
$$

where $\tilde{c}_{1}, \tilde{c}_{2} \in F^{*}\left(\mathbb{R}^{n}\right), \tilde{d}_{1}, \tilde{d}_{2} \in F^{*}\left(\mathbb{R}^{m}\right), \tilde{b}_{1} \in F^{*}\left(\mathbb{R}^{p}\right), \tilde{b}_{2} \in F^{*}\left(\mathbb{R}^{q}\right), \tilde{A}_{1}=\left(\tilde{a}_{i j}\right)_{p \times n}$, $\tilde{B}_{1}=\left(\tilde{b}_{i j}\right)_{p \times m}, \tilde{A}_{2}=\left(\tilde{e}_{i j}\right)_{q \times n}, \tilde{B}_{2}=\left(\tilde{s}_{i j}\right)_{q \times m}, \tilde{a}_{i}, \tilde{b}_{i}, \tilde{d}_{i}, \tilde{a}_{i j}, \tilde{b}_{i j}, \tilde{c}_{i j}, \tilde{s}_{i j} \in F^{*}(\mathbb{R})$.

For a dear understanding of the idea adopted, we define

$$
\left.\left.v_{1 \lambda_{j}}^{L-}=\frac{1}{2} \| c_{1 \lambda_{j}}^{L} x+d_{1 \lambda_{j}}^{L} y-g_{L \lambda_{j}}^{L}\right\}-\left(c_{1 \lambda_{j}}^{L} x+d_{1 \lambda_{3}}^{L} y-g_{L \lambda_{j}}^{L}\right)\right]
$$




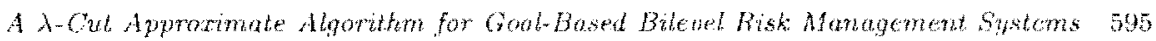

$$
\begin{aligned}
& v_{1 \lambda_{j}}^{L+}=\frac{1}{2}\left[\left|c_{1 \lambda_{j}}^{L} x+d_{1 \lambda_{j}}^{L} y-g_{L \lambda_{j}}^{L}\right|+\left(c_{1 \lambda_{j}}^{L} x+d_{1 \lambda_{j}}^{L} y-g_{L \lambda_{j}}^{L}\right)\right], \\
& v_{1 \lambda_{j}}^{R-}=\frac{1}{2}\left[\left|c_{1 \lambda_{j}}^{R} x+d_{1 \lambda_{j}}^{R} y-g_{L \lambda_{j}}^{R}\right|-\left(c_{1 \lambda_{j}}^{R} x+d_{1 \lambda_{j}}^{R} y-g_{L \lambda_{j}}^{R R}\right)\right], \\
& u_{1 \lambda_{2}}^{R+}=\frac{1}{2}\left[c_{1 \lambda_{j}}^{R} x+d_{\mid \lambda_{j}}^{R} y-g_{L \lambda_{j}}^{R} \mid+\left(c_{1 \lambda_{j}}^{R} x+d_{1 \lambda_{j}}^{R} y-g_{L \lambda_{j}}^{R}\right)\right] . \\
& v_{2 \lambda_{j}}^{L-}=\frac{1}{2}\left[\left|c_{2 \lambda_{j}}^{L} x+d_{2 \lambda_{j}}^{L} y-g_{F \lambda_{j}}^{L}\right|-\left(c_{2 \lambda_{j}}^{L} x+d_{2 \lambda_{j}}^{L} y-g_{F \lambda_{j}}^{L}\right)\right], \\
& v_{2 \lambda_{j}}^{L+}=\frac{1}{2}\left[\left|c_{2 \lambda_{j}}^{L} x+d_{2 \lambda_{j}}^{L} y-g_{1 \cdot \lambda_{j}}^{L}\right|+\left(c_{2 \lambda_{j}}^{L} x+d_{2 \lambda_{j}}^{L} y-g_{k \lambda_{j}}^{L}\right)\right], \\
& v_{2 \lambda_{j}}^{R-}=\frac{1}{2}\left[\left|c_{2 \lambda_{j}}^{R} x+d_{2 \lambda_{j}}^{R} y-g_{F \lambda_{j}}^{R}\right|-\left(c_{2 \lambda_{j}}^{R} x+d_{2 \lambda_{j}}^{R} y-g_{F \lambda_{j}}^{R}\right)\right], \\
& v_{2 \lambda_{j}}^{R+}=\frac{1}{2}\left[\left|r_{2 \lambda_{j}}^{R R} x+d_{2 \lambda_{j}}^{R} y-g_{F \lambda_{j}}^{R}\right|+\left(r_{2 \lambda_{j}}^{R} x+d_{2 \lambda_{j}}^{R} y-g_{r^{\prime} \lambda_{j}}^{R}\right)\right] .
\end{aligned}
$$

Associated with the FGBL problem defined by (7), we now consider the following bilevel decision problem:

For $\left(v_{1 \lambda_{j}}^{L-}, v_{1 \lambda_{j}}^{L+}, v_{1 \lambda_{j}}^{R-}, v_{1 \lambda_{j}}^{R+}\right) \in \mathbb{R}^{4} . X^{\prime} \subseteq X \times \mathbb{R}^{4},\left(v_{2 \lambda_{j}}^{L-} \cdot v_{2 \lambda_{j}}^{L+} \cdot v_{2 \lambda_{j}}^{R-}, v_{2 \lambda_{j}}^{R+}\right) \in \mathbb{R}^{4}$. $Y^{\prime} \subseteq Y \times \mathbb{R}^{4}$, let $x=\left(x_{1}, \ldots, x_{n}\right) \in X, x^{\prime}=\left(x_{1}, \ldots, x_{n_{2}}, v_{1 \lambda_{j}}^{L}, v_{1 \lambda_{j}}^{L+}, v_{1 \lambda_{j}}^{R}\right.$, $\left.v_{1 \lambda_{j}}^{R+}\right) \in X^{\prime}, y=\left(y_{1}, \ldots, y_{m}\right) \in Y \cdot y^{\prime}=\left(y_{1}, \ldots, y_{m}, v_{2 \lambda_{j}}^{L-}, v_{2 \lambda_{j}}^{L+}, v_{2 \lambda_{j}}^{R-}, v_{2 \lambda_{j}}^{R+}\right) \in Y^{\prime}$, and $v_{1 \lambda_{j}}^{L}, v_{1 \lambda_{j}}^{R}, v_{2 \lambda_{j}}^{L}, v_{2 \lambda_{j}}^{R}: X^{\prime} \times Y^{\prime \prime} \rightarrow F^{*}(\mathbb{R})$.

$$
\begin{aligned}
& \min _{x^{\prime} \in X^{\prime}} v_{1 \lambda_{j}}^{L}=v_{1 \lambda_{j}}^{L-}+v_{1 \lambda_{j}}^{L+}, \\
& \min _{x_{i}^{\prime} \in X^{\prime}} v_{1 \lambda_{i}}^{R}=v_{1 \lambda_{j}}^{R-}+v_{1 \lambda_{j}}^{R+} \text {. } \\
& \text { subject to } c_{1 \lambda_{j}}^{L} x+d_{1 \lambda_{j}}^{L} y+v_{1 \lambda_{j}}^{L-}-v_{1 \lambda_{j}}^{L+}=g_{L \lambda_{j}}^{L} \text {, } \\
& c_{1 \lambda_{j}}^{R} x+d_{1 \lambda_{2}}^{R} y+v_{1 \lambda_{j}}^{R-}-v_{1 \lambda_{j}}^{R++}=g_{l \lambda_{1}}^{R}, \\
& v_{1 \lambda_{j}}^{L, \cdots}, v_{1 \lambda_{j}}^{L+}, v_{1 \lambda_{j}}^{R-} \cdot v_{1 \lambda_{j}}^{R+} \geq 0 \text {, } \\
& v_{1 \lambda_{2}}^{L-w} \cdot v_{1 \lambda_{j}}^{L+}=0 \\
& v_{1 \lambda_{3}}^{R-} \cdot v_{1 \lambda_{3}}^{R+\lambda_{j}}=0
\end{aligned}
$$

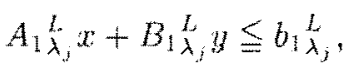

$$
\begin{aligned}
& A_{1, \lambda_{3}}^{R} x+B_{1 \lambda_{2} y}^{R} y \leqq b_{1}^{R} \text {. } \\
& \min _{y^{\prime} \in Y^{\prime}} v_{2 \lambda_{j}}^{L}=v_{2 \lambda_{j}}^{L-}+v_{2 \lambda_{j}}^{L+} \text {; } \\
& \min _{u^{\prime} \in Y^{\prime}, 2 \lambda_{j}} v^{R}=l_{2 \lambda_{j}}^{R-}+v_{2 \lambda_{j}}^{R+} \text {. }
\end{aligned}
$$




$$
\begin{aligned}
& \text { subject to } c_{2 \lambda_{1}}^{L} x+d_{2 \lambda_{3}}^{L} y+v_{2 \lambda_{3}}^{L-\alpha}-u_{2 \lambda_{3}}^{L+}=g_{F \lambda_{1}}^{L} \text {, } \\
& c_{2 \lambda_{3}}^{R} l+d_{2 \lambda_{3}}^{R} y+v_{3 \lambda_{3}}^{R-}-v_{2 \lambda_{1}}^{R+}=g_{k \lambda_{j}}^{R} . \\
& v_{2 \lambda_{j}}^{L-}, v_{2 \lambda_{j}}^{L+} \cdot v_{2 \lambda_{j}}^{R-}, v_{2 \lambda_{j}}^{R+} \geq 0, \\
& v_{2 \lambda_{j}}^{L-L_{j}} \cdot v_{2 \lambda_{j}}^{L++}=0 \\
& v_{2 \lambda_{j}}^{R-} \cdot v_{2 \lambda_{j}}^{R+}=0 \\
& A_{2 \lambda_{j}}^{L} x+B_{2 \lambda_{j}}^{L} y \leqq b_{2} L, \\
& A_{2}{ }_{2}^{R} t+B_{2 \lambda_{j}}^{R} y \leqq b_{2 \lambda_{j}}^{k}, \\
& j=0,1.2 \ldots \ldots l \text {. }
\end{aligned}
$$

Theorem 3.1. Let $\left(x^{\prime *} \cdot y^{\prime *}\right)=\left(x^{*}, v_{1 \lambda_{j}}^{L \cdots *}, v_{1 \lambda_{j}}^{L+*}, v_{1 \lambda_{j}}^{R+*}, v_{2 \lambda_{3}}^{R+*}, y^{*}, v_{2 \lambda_{j}}^{L-\cdots *} \cdot v_{2 \lambda_{j}}^{L+*}, v_{2 \lambda_{j}}^{R-\cdots *}\right.$. $\left.v_{2 \lambda_{i}}^{R+*}\right)$ be the optimal solution to the bilend decision problem $(9)$ : then, $\left(x^{*}, y^{*}\right)$ is the optimal solution to the bilevel decision problem defined by (7).

Proof. By Definition 3.2, let the notations associated with problem (7) be denoted by

$$
\begin{aligned}
& S=\left\{(x, y): A_{i \lambda_{j}}^{L} x+B_{i} \stackrel{L}{L} y \leqq b_{i \lambda_{j}}^{L}, A_{i \lambda_{j}}^{R} x+B_{i \lambda_{j}}^{R} y \leqq u_{i \lambda_{j}}^{R},\right. \\
& i=1,2 \cdot j=0,1,2, \ldots, l\}, \\
& S(X)=\left\{x \in X: \exists y \in Y, A_{i} \lambda_{j} x+B_{i \lambda_{j}}^{L} y \leqq b_{i \lambda_{j}}^{L}, A_{i \lambda_{j}}^{R} x+B_{i, \lambda_{j}}^{R} y \leqq b_{i \lambda_{1}}^{R}\right. \text {, } \\
& i=1.2, j=0,1,2 \ldots, l\} \\
& S(x)=\{y \in Y:(x, y) \in S\} \text {, } \\
& P(x)=\left\{y \in Y: y \in \operatorname{argmin}\left|c_{2 \lambda_{j}}^{L} x+d_{2 \lambda_{j}}^{L} \dot{y}-g_{F \lambda_{j}}^{L}\right| .\right. \\
& \left.\left|c_{2 \lambda_{j}}^{R} x+d_{2 \lambda_{j}}^{R} y-g_{i \lambda_{j}}^{R}\right|: \ddot{y} \in S(x) \mid\right\} \text { : } \\
& I R=\{(x, y):(x, y) \in S, y \in P(x)\} .
\end{aligned}
$$

Problem (7) can be written as

$$
\min \left\{\left|c_{1 \lambda_{j}}^{L} x+d_{1 \lambda_{j}}^{L} y-g_{L \lambda_{j}}^{L}\right| \cdot\left|c_{1 \lambda_{j}}^{R} x+d_{1 \lambda_{j}}^{R} y-g_{L \lambda_{j}}^{R}\right|:(x, y) \in I R\right\} .
$$

and those of problem (9) are denoted by

$$
\begin{aligned}
S^{\prime}= & \left\{\left(x^{\prime}, y^{\prime}\right): A_{i \lambda_{j}}^{L} x+B_{i \lambda_{j}}^{L} y \leqq b_{i \lambda_{j}}^{L}, A_{i \lambda_{j}}^{R} x+B_{i \lambda_{j}}^{R} y \leqq b_{i \lambda_{j}}^{R},\right. \\
& v_{i \lambda_{j}}^{L-} \cdot v_{i \lambda_{j}}^{L+}=0, v_{i \lambda_{j}}^{R-} \cdot v_{i \lambda_{j}}^{R+}=0, i=1,2, \\
& c_{1 \lambda_{j}}^{L} x+d_{i \lambda_{j}}^{L} y+v_{1 \lambda_{j}}^{L-}-v_{1 \lambda_{j}}^{L+}=g_{L \lambda_{j}}^{L}, c_{1 \lambda_{j}}^{R} x+d_{i \lambda_{j}}^{R} y+v_{1 \lambda_{j}}^{R-}-v_{1 \lambda_{j}}^{R+}=g_{L \lambda_{j}}^{R}, \\
& c_{2 \lambda_{j}}^{L} x+d_{2 \lambda_{j}}^{L} y+v_{2 \lambda_{j}}^{L-}-v_{2 \lambda_{j}}^{L+}=g_{F \lambda_{j}}^{L}, c_{2 \lambda_{j}}^{R} x+d_{2 \lambda_{j}}^{R} y+v_{2 \lambda_{j}}^{R-}-v_{2 \lambda_{j}}^{R+}=g_{F \lambda_{j}}^{R}, \\
& j=0.1 .2, \ldots, l\},
\end{aligned}
$$




$$
\begin{aligned}
& S\left(X^{\prime}\right)=\left\{x^{\prime} \in X^{\prime}: \exists y^{\prime} \in Y^{\prime \prime} \cdot A_{i \lambda_{j}}^{l} x+B_{i \lambda_{j}}^{L} y \leqq b_{i \lambda_{j}}^{L}, A_{i \lambda_{j}}^{R} x+B_{i \lambda_{j}}^{R} y \leqq b_{i \lambda_{j}}^{R} .\right.
\end{aligned}
$$

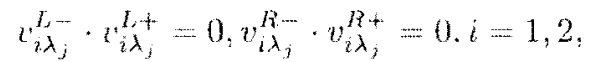

$$
\begin{aligned}
& c_{1 \lambda_{j}}^{L} x+d_{i \lambda_{j}}^{L} y+v_{1 \lambda_{j}}^{L-}-v_{1 \lambda_{j}}^{L+}=g_{L \lambda_{j}}^{L}, c_{1 \lambda_{j}}^{R} x+d_{i \lambda_{j}}^{R} y+v_{1 \lambda_{j}}^{R-}-v_{1 \lambda_{j}}^{R+}=g_{L \lambda_{j}}^{R}, \\
& c_{2 \lambda_{j}}^{L} x+d_{2 \lambda_{j}}^{L} y+v_{2 \lambda_{j}}^{L-}-v_{2 \lambda_{j}}^{L+}=g_{F \lambda_{j}}^{L}, c_{2 \lambda_{j}}^{R} x+d_{2 \lambda_{j}}^{R} y+v_{2 \lambda_{j}}^{R-}-v_{2 \lambda_{j}}^{R+}=g_{F \lambda_{j}}^{R} . \\
& j=0.1 \ldots, l\} \text {. } \\
& S\left(x^{\prime}\right)=\left\{y^{\prime} \in Y^{\prime \prime}:\left(x^{\prime}, y^{\prime}\right) \in S^{\prime}\right\}, \\
& P\left(x^{\prime}\right)=\left\{y^{\prime} \in Y^{\prime}: y^{\prime} \in \operatorname{argmin}\left[\hat{v}_{2 \lambda_{j}}^{L-}+i_{2 \lambda_{j}}^{L+} \cdot v_{2 \lambda_{j}}^{R-}+v_{2 \lambda_{j}}^{R+}: \ddot{y}^{\prime} \in S\left(x^{\prime}\right)\right]\right\}, \\
& I R^{\prime}=\left\{\left(x^{\prime}, y^{\prime}\right):\left(x^{\prime}, y^{\prime}\right) \in S^{\prime}, y^{\prime} \in P\left(x^{\prime}\right)\right\} .
\end{aligned}
$$

Problem (9) can be written as

$$
\min \left\{v_{1 \lambda_{j}}^{L-}+v_{1 \lambda_{j}}^{L+}, v_{1 \lambda_{j}}^{R--}+v_{1 \lambda_{j}}^{R+}:\left(x^{\prime}, y^{\prime}\right) \in I R^{\prime}\right\}
$$

As $\left(x^{* *}, y^{\prime *}\right)$ is the optimal solution to the problem (9), from (13), it can be

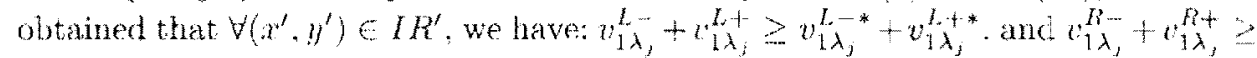
$v_{1 \lambda_{i}}^{R-*}+v_{1 \lambda_{i}}^{R+*}$

As

$$
c_{1 \lambda_{j}}^{L} x+d_{1 \lambda_{j}}^{L} y+v_{1 \lambda_{j}}^{L-}-v_{1 \lambda_{j}}^{L++}=g_{L \lambda_{j}}^{L \lambda_{j}} \text { and } v_{1 \lambda_{j}}^{L-} \cdot c_{1 \lambda_{j}}^{L+}=0
$$

we have

$$
\begin{aligned}
& v_{1 \lambda_{j}}^{L-}+v_{1 \lambda_{j}}^{L+}=\left|c_{1 \lambda_{j}}^{L} x+d_{1 \lambda_{j}}^{L} y-g_{L \lambda_{j}}^{L}\right|, \quad \text { and } \\
& v_{1 \lambda_{j}}^{L-*}+v_{1 \lambda_{j}}^{L+*}=\left|c_{1 \lambda_{j}}^{L} x^{*}+d_{1 \lambda_{j}}^{L} y^{*}-g_{L \lambda_{j}}^{L}\right| .
\end{aligned}
$$

So,

$$
\left|c_{1 \lambda_{j}}^{L} x+d_{1 \lambda_{j}}^{L} y-g_{L \lambda_{j}}^{L}\right| \geq\left|c_{1 \lambda_{j}}^{L} x^{*}+d_{1 \lambda_{j}}^{L} y^{*}-g_{L \lambda_{j}}^{L}\right|
$$

Similarly, we can get that

$$
\left|c_{1 \lambda_{i}}^{R} x+d_{1 \lambda_{j}}^{R} y-g_{L \lambda_{j}}^{R}\right| \geq\left|c_{1 \lambda_{j}}^{R} x^{*}+d_{1 \lambda_{j}, y^{*}}^{R}-g_{L \lambda_{j}}^{R}\right| .
$$

Now we prove that the projection of $S^{\prime}$ onto the $X \times Y$ space denoted by $S^{\prime} \mid x, r$, is equal to $S$.

On the one hand. $\left.\forall(x, y) \in S^{\prime}\right|_{x . Y}$. from constraints: $A_{2}^{l}{ }_{\lambda_{i}}^{L} x+B_{i}{ }_{\lambda_{i}}^{L} y \leq$ $b_{i \lambda_{j}}^{L}, A_{i}{ }_{\lambda_{j}}^{R} x+B_{i \lambda_{j}}^{R} y \leqq b_{i \lambda_{j}}^{R}, i=1.2$ in $S^{\prime}$, we have: $(x, y) \in S$, so $S^{\prime} \mid x . \subseteq S$.

On the other hand, $\forall(x, y) \in S$, by (8). we can always find such $v_{i \lambda_{j}}^{L-}, v_{i \lambda_{j}}^{L+}, v_{i \lambda_{j}}^{R-}, v_{i \lambda_{j}}^{R+}, i=1,2$, which make constraints: $v_{i \lambda_{j}}^{R-} \cdot v_{i \lambda_{j}}^{R+}=0, i=1,2$, $c_{1 \lambda_{j}}^{L} x+d_{i \lambda_{j}}^{L} y+v_{1 \lambda_{j}}^{L-}-v_{1 \lambda_{j}}^{L+}=g_{L \lambda_{j}}^{L}, c_{1 \lambda_{j}}^{R} x+d_{i \lambda_{j}}^{R} y+v_{1 \lambda_{j}}^{R-}-v_{1 \lambda_{j}}^{R+}=g_{L \lambda_{j}}^{R} \cdot c_{2 \lambda_{j}}^{L} x+$ $d_{2 \lambda_{j}}^{L} y+v_{2 \lambda_{j}}^{L-}-v_{2 \lambda_{j}}^{L+}=g_{F \lambda_{j}}^{L}$ and $c_{2 \lambda_{j}}^{R} x+d_{2 \lambda_{3}}^{R} y+v_{2 \lambda_{j}}^{R-}-v_{2 \lambda_{j}}^{R+}=g_{F^{\prime} \lambda_{j}}^{R}$ satisfied. Together 
with the inequations of $A_{i}{ }_{\lambda_{j}}^{L} x+B_{i \lambda_{j}}^{L} y \leqq b_{i \lambda_{j}}^{L}$ and $A_{i \lambda_{i}}^{R} x+B_{i \lambda_{j}}^{R} y \leqq b_{i \lambda_{j}}^{R}, i=1,2$ requested by $S$, we have $\left(x, v_{1 \lambda_{j}}^{L-}, v_{1 \lambda_{j}}^{L+}, y, v_{2 \lambda_{j}}^{R-}, v_{2 \lambda_{j}}^{R+}\right) \in S^{\prime}$ : thus, $\left.(x, y) \in S^{\prime}\right|_{X, Y}$, $S \subseteq S^{\prime} \mid X, Y$.

So, we can prove that

$$
S^{\prime} \mid X Y=S
$$

Similarly. we have

$$
\begin{aligned}
& \left.S(x)^{\prime}\right|_{X, Y}=S(x) \\
& \left.S(X)^{\prime}\right|_{X, Y}=S(X)
\end{aligned}
$$

Also, from

$$
c_{3 \lambda_{j}}^{L} x+d_{2 \lambda_{j}}^{L} y+v_{2 \lambda_{j}}^{L--}-v_{2 \lambda_{j}}^{L+}=g_{F^{2} \lambda_{j}}^{L} \text { and } v_{2 \lambda_{j}}^{L-} \cdot v_{2 \lambda_{j}}^{L+}=0 .
$$

we have

$$
v_{2 \lambda_{j}}^{L-}+v_{2 \lambda_{3}}^{L+}=\left|c_{2 \lambda_{j}}^{L} x+d_{2 \lambda_{j}}^{L} y-g_{F \lambda_{j}}^{L}\right| .
$$

Similarly, we have

$$
v_{2 \lambda_{j}}^{R-}+v_{2 \lambda_{j}}^{R+}=\left|c_{2 \lambda_{j}}^{R} x+d_{2 \lambda_{j}}^{R} y-g_{F \lambda_{j}}^{R}\right|
$$

Thus,

$$
\begin{aligned}
P\left(x^{\prime}\right)= & \left\{y^{\prime} \in Y^{\prime}: y^{\prime} \in \operatorname{argmin}\left[c_{2 \lambda_{j}}^{L} x+d_{2 \lambda_{j}}^{L} y-g^{L} \lambda_{\lambda_{j}}\right] .\right. \\
& \left.\left.\left|c_{2 \lambda_{j}}^{R} x+d_{2 \lambda_{j}}^{R} \dot{y}-g_{F \lambda_{y}}^{R}\right|: \ddot{y}^{\prime} \in S\left(x^{\prime}\right)\right]\right\} .
\end{aligned}
$$

From (15) and (18), we get

$$
P\left(x^{\prime}\right) \mid x \times Y=P(x) .
$$

From (10e), (12e), (15), and (19), we get

$$
\left.I R^{\prime}\right|_{X \times Y}=I R,
$$

which means, the leaders of (7) and (9) share the same optimizing space in $X \times Y$ space.

Thus, from (14) and (20) and the discussions above, we have

$\forall(x, y) \in I R$, we have $\left|c_{1 \lambda_{j}}^{L} x+d_{1, \lambda_{3}}^{L} y-g_{L \lambda_{j}}^{L}\right| \geq\left|c_{1 \lambda_{2}}^{L} x^{*}+d_{\mid \lambda_{1}}^{L} y^{*}-g_{1, \lambda_{1}}^{L}\right| \cdot \mid c_{\lambda_{\lambda_{j}}}^{R} x+$ $d_{1 \lambda_{j}}^{R} y-g_{L \lambda_{j}}^{R}|\geq| e_{1 \lambda_{3}}^{R} x^{*}+d_{1 \lambda_{3}}^{R} y^{*}-g_{L \lambda_{j}}^{R}$

So. $\left(x^{*}, y^{*}\right)$ is the optimal solution of problem (7).

By adopting weighting method, (9) can be further transforred into (21):

$$
\min _{x^{\prime} \in X^{\prime}} v_{1 \lambda_{j}}^{L-}+v_{1 \lambda_{j}}^{L++}+v_{1 \lambda_{j}}^{R-}+v_{1 \lambda_{j}}^{R+}
$$




$$
\begin{aligned}
& \text { subject to } c_{1 \lambda_{j}}^{L} x+d_{1 \lambda_{j}}^{L} y+v_{1 \lambda_{j}}^{L-}-v_{1 \lambda_{j}}^{L+}=g_{L \lambda_{j}}^{L} \text {. } \\
& c_{1 \lambda_{j}}^{R} x+d_{1 \lambda_{j}}^{R} y+v_{1 \lambda_{j}}^{R \cdots}-v_{1 \lambda_{j}}^{R+}=g_{L \lambda_{j}}^{R} . \\
& v_{1 \lambda_{j}}^{L \cdots} \cdot u_{1 \lambda_{j}}^{L+}, v_{1 \lambda_{2}}^{R-\cdots}, v_{1 \lambda_{1}}^{R+} \geq 0 \\
& v_{1 \lambda_{2}}^{L-} \cdot v_{1 \lambda_{3}}^{L++}=0 \\
& n_{1 \lambda_{j}}^{R-} \cdot l_{1 \lambda_{j}}^{R+}=0 \\
& A_{1}{ }_{\lambda_{j}}^{L} x+B_{1 \lambda_{j}}^{L} y \leq b_{1 \lambda_{i}}^{L} \text {. } \\
& A_{1} \lambda_{\lambda_{3}}^{R} x+B_{1}^{R} \lambda_{j}^{R} y \leqq b_{1}^{R}, \\
& \min _{y^{\prime} \in \bar{Y}^{\prime \prime}} v_{2 \lambda_{j}}^{L-}+v_{2 \lambda_{j}}^{L+}+v_{2 \lambda_{j}}^{R-}+v_{2 \lambda_{j}}^{R+} \text {, } \\
& \text { subject to } c_{2 \lambda_{j}}^{L} x+d_{2 \lambda_{j}}^{L} y+t_{2 \lambda_{\lambda}}^{L-}-i_{2 \lambda_{j}}^{L+}=g_{* \lambda_{j}}^{L} \text {. } \\
& c_{2 \lambda_{j}}^{R} x+d_{2 \lambda_{j}}^{R} y+v_{2 \lambda_{j}}^{R-}-v_{2 \lambda_{j}}^{R+}=g_{F \lambda_{j}}^{R} . \\
& v_{2 \lambda_{j}}^{L}, v_{2 \lambda_{j}}^{L+}, v_{2 \lambda_{j}}^{R-}, v_{2 \lambda_{j}}^{R+} \geq 0 \\
& v_{2 \lambda_{j}}^{L-} \cdot v_{2 \lambda_{j}}^{L+}=0 \\
& v_{2 \lambda_{j}}^{R-} \cdot v_{2 \lambda_{i}}^{R+}=0 \\
& A_{2 \lambda_{1}}{ }^{L} x+B_{2} \lambda_{3} y \leqq b_{2}{ }_{\lambda_{3}}, \\
& A_{2}{ }_{\lambda_{3}}^{R} x+B_{2}{ }_{\lambda_{3}}^{R} y \leqq b_{2} \lambda_{3}, \\
& j=0.1, \ldots, l \text {. }
\end{aligned}
$$

The nonlinear conditions of $v_{i \lambda_{j}}^{L--} \cdot v_{i \lambda_{j}}^{L+}=0$ and $v_{i \lambda_{j}}^{R--} \cdot v_{i \lambda_{j}}^{R+}=0, i=1,2$ need not be maintained if the Kuhn- ${ }^{-}$Tucker approach ${ }^{2 \cdot 3}$ together with Simplex algorithm are adopted. since only equivalence at an optimum is wanted. Further explanation can be found from Ref. 4. Thus, problem (21) is further transfomed as follows:

For $\left(v_{1 \lambda_{j}}^{-}, v_{1 \lambda_{j}}^{+}\right) \in \mathbb{R}^{2}, \bar{X}^{\prime} \subseteq X \times \mathbb{R}^{2},\left(v_{2 \lambda_{j}}^{-}, v_{2 \lambda_{j}}^{+}\right) \in \mathbb{R}^{2}, \bar{Y}^{\prime} \subseteq Y \times \mathbb{R}^{2}$; let $x=\left(x_{1}, \ldots, x_{n}\right) \in X, \bar{x}^{\prime}=\left(x_{1}, \ldots, x_{n}, v_{1 \lambda_{j}}^{-}, v_{1 \lambda_{j}}^{+}\right) \in X^{\prime}, y=\left(y_{1}, \ldots, y_{n}\right) \in Y$, $\bar{y}^{\prime}=\left(y_{1}, \ldots, y_{n}, v_{2 \lambda_{j}}^{-}, v_{2 \lambda_{j}}^{+}\right) \in \bar{Y}^{\prime}$. and $v_{1 \lambda_{j}}, v_{2 \lambda_{j}}: \bar{X}^{\prime} \times \bar{Y}^{\prime} \rightarrow F^{*}(\mathbb{R})$.

$$
\begin{aligned}
& \min _{y^{\prime} \in \bar{X}^{\prime}} v_{\lambda \lambda_{j}}=v_{\overline{1} \lambda_{j}}^{-}+z_{1 \lambda_{j}}^{+} \\
& \text {subject to }\left(c_{1 \lambda_{j}}^{L}+c_{1 \lambda_{j}}^{R}\right) x+\left(d_{1 \lambda_{j}}^{L}+d_{1 \lambda_{j}}^{R}\right) y+v_{1 \lambda_{j}}^{-}-v_{1 \lambda_{1}}^{+}=g_{\lambda_{\lambda_{j}}}^{L}+g_{L \lambda_{j}}^{R},
\end{aligned}
$$

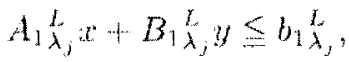

$$
\begin{aligned}
& A_{1 \lambda_{j}}^{R} x+B_{1 \lambda_{j}}^{R} y \leqq b_{1 \lambda_{j}}^{R}, \\
& \operatorname{limin}_{y^{\prime} \in Y,} v_{2 \lambda_{j}}=v_{2 \lambda_{j}}^{-}+v_{2 \lambda_{j}}^{+}
\end{aligned}
$$




$$
\begin{array}{rl}
\text { subject to } & \left(c_{2 \lambda_{j}}^{L}+c_{2 \lambda_{j}}^{R}\right) x+\left(d_{2 \lambda_{j}}^{L}+d_{2 \lambda_{j}}^{R}\right) y+v_{2 \lambda_{j}}^{-}-u_{2 \lambda_{j}}^{+}=g_{F \lambda_{j}}^{L}+g_{k \lambda_{j}}^{R}, \\
& A_{2 \lambda_{j}}^{R} x+B_{2 \lambda_{j}}^{L} y \leqq b_{2 \lambda_{j}}^{L} . \\
& A_{2 \lambda_{j}}^{R} x+B_{2 \lambda_{j}}^{R} y \leqq b_{2 \lambda_{j}}^{R}, \\
j & j=0,1, \ldots, l
\end{array}
$$

where $v_{i \lambda_{j}}^{\cdots-\lambda_{j}}=v_{i \lambda_{j}}^{L--}+v_{i \lambda_{j}}^{R--}, v_{i \lambda_{j}}^{++}=v_{i \lambda_{j}+}^{L+}+v_{i \lambda_{j}}^{R+}, i=1.2$.

Problem (22) is a standard linear bilevel decision problem, which can be solved by Kuhn Tucker approach. ${ }^{23}$

Based on the discussions above, the $\lambda$-cut approximate algorithm for solving the FGBL problens is detailed as follows:

Step 1 (Input) Get relevant coefficients of a FGBL problem which inchude:

(1) Coefficients of (1)

(2) Coefficients of $\tilde{g}_{L}$ and $\tilde{g}_{F}$

(3) Satisfactory degree: $\alpha$

(4) $\varepsilon>0$

Step 2 (Initializing) Let $k=1$, which is the connter to record current loop.

In (7), where $\lambda_{j} \in[\alpha, 1]$. let $\lambda_{0}=\alpha$ and $\lambda_{1}=1$. respectively; then, each objective will be transferred into four nonfuzzy objective functions, and each finzzy constraint is converted into four nonfuzzy constraints.

Step 3 (Computing) By introducing auxiliary variables $c_{i \lambda_{2}}$ and $v_{2 \lambda_{j}}^{++}, i=1,2$. we get the format of (22).

The solution $\left(x, v_{1 \lambda_{1}}^{-}, v_{2 \lambda_{j}}^{+}, y, v_{2 \lambda_{1}}^{-}, v_{2 \lambda_{3}}^{+}\right)_{2}$ of $(22)$ is abtained by Kuhn Thicker approach.

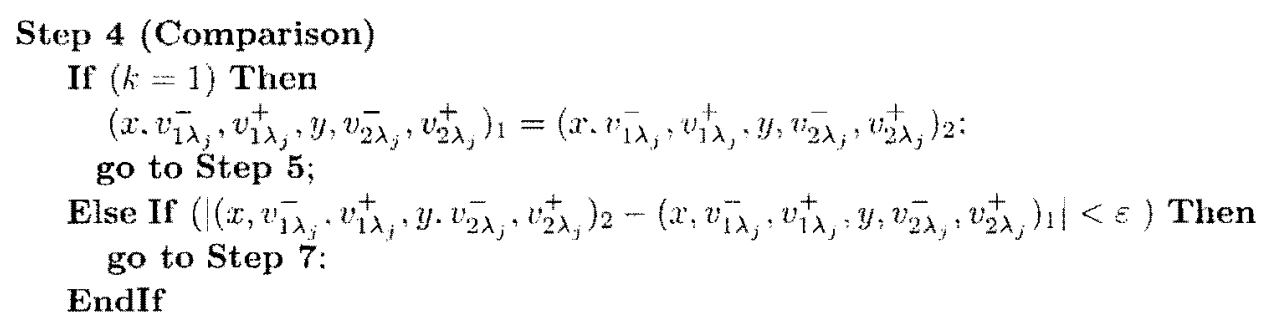

Step 5 (Splitting) Suppose there are $(L+1)$ nodes $\lambda_{j}(j=0.2,4, \ldots, 2 L)$ in the interval $[\alpha, 1]$, insert $L$ new nodes $\lambda_{j}(j=1,3, \ldots, 2 L-1)$, which satisty

$$
\lambda_{2 j+1}=\left(\lambda_{2 j}+\lambda_{2 j+2}\right) / 2 . \quad(j=0,1,2 \ldots, L-1) .
$$




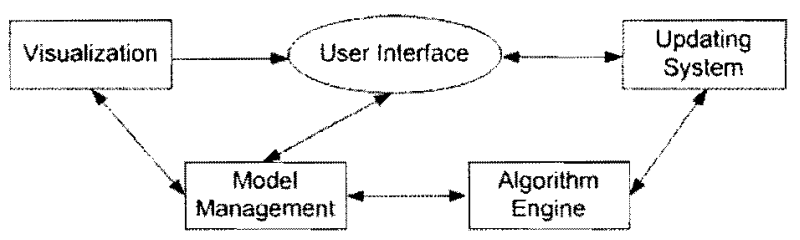

Fig. 1. The system structure of the FGBL software system.

\section{Step 6 (Loop) \\ $k=k+1$ \\ go to Step 3;}

Step 7 (Output) $(x . y)_{2}$ is obtained as a final solution.

To realize this algorithm proposed above, a FGBL software system is developed using Visual Basic 6.0. This FGBL software system provides computerized assistance to decision makers in a decentratized organization to gather knowledge about a FGBL problem and controls the decision-making process for a better-informed decision.

The structure of the software system is depicted in Fig. 1. Within this architecture, five modules are involved, i.e. "user interface," "model management," "algorithm engine," "updating system," and "visualization." Data are collected through user interface and formatted as a FGBL model by model management module. The core calculations are carried in algorithm engineer owcr a FCBL model, and the solution is output through visualization module to an end user by user interface.

\section{Examples}

This section employs a mumerical example and a case-bascd example to show the running procedure and the application of the proposed algorithm.

\subsection{A numerical example}

We first use the proposed algorithm to solve a numerical FGBL problem.

Step 1 (Input relevant coefficients)

(1) Coefficients of (1):

$$
\begin{aligned}
& \max _{x \in X} F(x, y)=\tilde{c}_{1} x+\tilde{d}_{1} y, \\
& \text { subject to } \tilde{A}_{1} x+\tilde{B}_{1} y \leq \tilde{b_{1}}, \\
& \min _{y \in Y} f(x, y)=\tilde{c}_{2} x+\tilde{d}_{2} y . \\
& \text { subject to } \tilde{A}_{2} x+\tilde{B}_{2} y \leq \tilde{b_{2}},
\end{aligned}
$$

where $x \in \mathbb{R}, y \in \mathbb{R}$, and $X=x \geq 0, Y=y \geq 0$. 
The menbership functions of the coefficients of the objectire functions and the constraints of both the leader and the follower are as follows:

$$
\begin{aligned}
& \mu_{\bar{c}_{1}}(x)=\left\{\begin{array}{ll}
0, & x<5, \\
\left(x^{2}-25\right) / 11 . & 5 \leqq x<8, \\
1, & x=6, \\
\left(64-x^{2}\right) / 28, & 6<x \leq 8 . \\
0 . & x>8,
\end{array} \quad \mu_{\bar{d}_{1}}(x)= \begin{cases}0, & x<2, \\
\left(x^{2}-4\right) / 5, & 2 \leq x<3 \\
1 . & x=3, \\
\left(25-x^{2}\right) / 16, & 3<x \leq 5 \\
0, & x>5\end{cases} \right. \\
& \mu_{\tilde{r}_{2}}(x)=\left\{\begin{array}{ll}
0, & x<-4 . \\
\left(16-x^{2}\right) / 7, & -4 \leqq x<-3, \\
1, & x=-3, \\
\left(x^{2}-1\right) / 8, & -3<x \leq-1 . \\
0, & x>-1 .
\end{array} \quad \mu_{\tilde{d}_{2}}(x)= \begin{cases}0 . & x<5 \\
\left(x^{2}-25\right) / 11, & 5 \leqq x<6 \\
1, & x=6 \\
\left(61-x^{2}\right) / 28, & 6<x \leq 8 \\
0, & x>8\end{cases} \right. \\
& \mu_{\bar{A}_{1}}(x)= \begin{cases}0, & x<-2 \\
\left(4-x^{2}\right) / 3, & -2 \leq x<-1 \\
1, & x=-1 \\
\left(x^{2}-0.25\right) / 0.75, & -\cdots 1<x \leq-0.5 \\
0, & x>-0.5\end{cases} \\
& \mu_{\dot{B}_{1}}(x)= \begin{cases}0, & x<2 \\
\left(x^{2}-4\right) / 5, & 2 \leq x<3 \\
1, & x=3 \\
\left(25-x^{2}\right) / 16, & 3<x \leq 5 \\
0 . & x>5\end{cases} \\
& \mu_{\ddot{A}_{2}}(x)= \begin{cases}0, & x<0.5 \\
\left(x^{2}-0.25\right) / 0.75, & 0.5 \leq x<1 . \\
1, & x=1, \\
\left(4-x^{2}\right) / 3, & 1<x \leq 2, \\
0, & x>2\end{cases} \\
& \mu_{\mathcal{B}_{2}}(x)= \begin{cases}0, & x<2 \\
\left(x^{2}-4\right) / 5, & 2 \leq x<3 \\
1, & x=3 \\
\left(25-x^{2}\right) / 16, & 3<x \leq 5 \\
0, & x>5\end{cases}
\end{aligned}
$$




$$
\begin{aligned}
& \mu_{\tilde{h}_{1}}(x)= \begin{cases}0, & x<19 \\
\left(x^{2}-361\right) / 80, & 19 \leqq x<21 \\
1 . & x=21 \\
\left(625-x^{2}\right) / 184, & 21<x \leq 25 \\
0, & x>25\end{cases} \\
& \mu_{\tilde{b}_{3}}(x)= \begin{cases}0, & x<25 \\
\left(x^{2}-625\right) / 104, & 25 \leqq x<27, \\
1, & x=27, \\
\left(961-x^{2}\right) / 232, & 27<x \leq 31 \\
0, & x>31\end{cases}
\end{aligned}
$$

(2) The membership functions for the fitzoy goals of $\tilde{g}_{L}$ and $\tilde{g}_{F}$ are

$$
\begin{aligned}
& H_{\dot{G}_{L}}(x)= \begin{cases}0, & x<15, \\
\left(x^{2}-225\right) / 175, & 15 \leq x<20, \\
1, & x=20, \\
\left(900-x^{2}\right) / 500, & 20<x \leq 30, \\
0, & x>30,\end{cases} \\
& \mu_{\bar{y} F}(x)= \begin{cases}0, & x<4, \\
\left(x^{2}-16\right) / 48, & 4 \leq x<8, \\
1, & x=8, \\
\left(225-x^{2}\right) / 161, & 8<x \leq 15, \\
0, & x>15 .\end{cases}
\end{aligned}
$$

(3) Satisfactory degree: $\alpha=0.2$

(4) $\varepsilon=0.01$.

Step 2 (Initializing) Let $k=1$. Associated with this example, the corresponding $\lambda$-cut set based FGBL problem is

$$
\begin{aligned}
& \min _{x \in X}|\sqrt{11 \lambda+25} x+\sqrt{5 \lambda+4} y-\sqrt{175 \lambda+225}| \\
& \min _{x \in X}|\sqrt{64-28 \lambda} x+\sqrt{25-16 \lambda} y-\sqrt{900-500 \lambda}|
\end{aligned}
$$




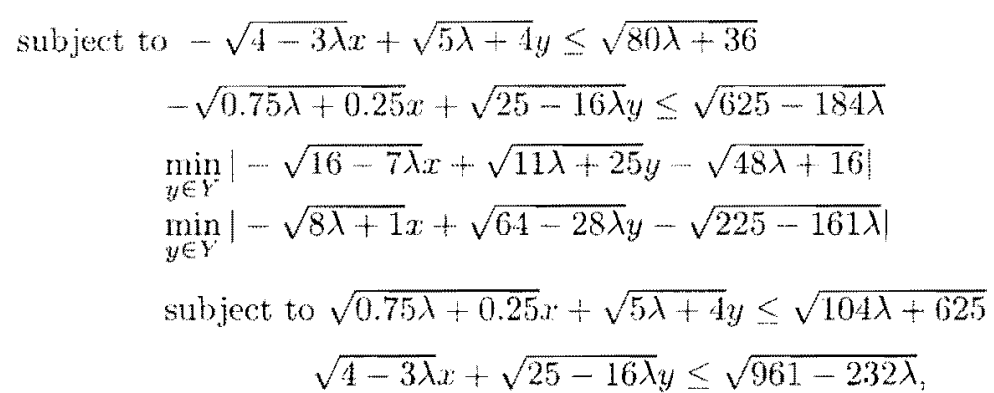

where $\lambda \in[0.2,1]$.

Referring to the algoritlim, only $\lambda_{\theta}=0.2$ and $\lambda_{1}=1$ are considered initially, Thus, four nonfuzzy objective functions and four nonfuzzy constraints for the leader and follower are generated, respectively:

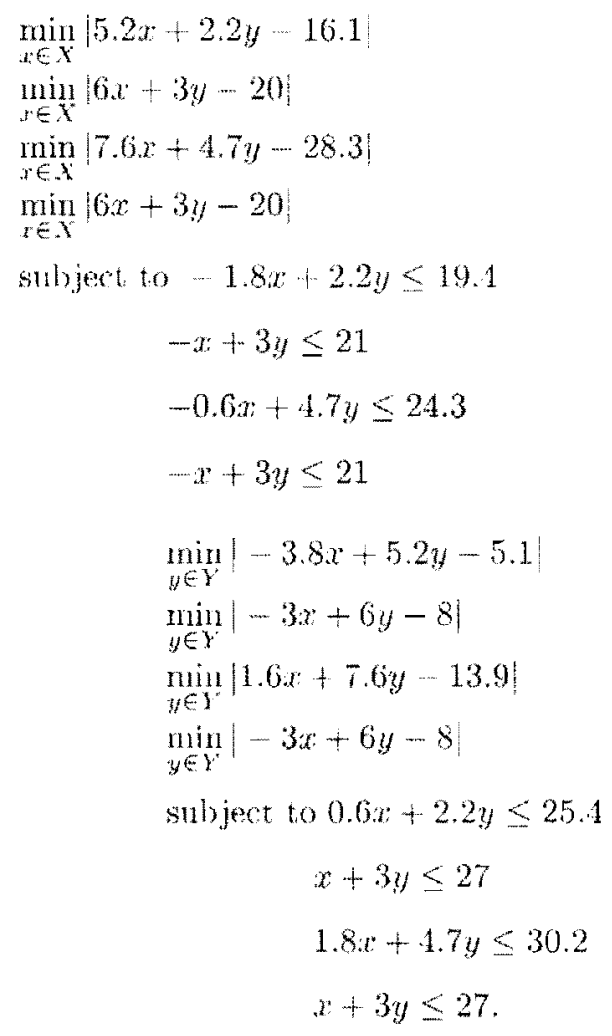

Step 3 (Computing) By introducing aluxiliary variables $v_{i}^{-}, v_{i}^{+}, i=1,2$, we get $\min _{\left(x, v_{1}^{-}, v_{1}^{+}\right) \in X^{,}} v_{1}^{-}+v_{1}^{+}$ 


$$
\begin{aligned}
& \text { subject to } 24.8 x+12.9 y+v_{1}^{-}-v_{1}^{+}=84.4, \\
& -1.8 x+2.2 y \leq 19.4 . \\
& -x+3 y \leq 21, \\
& -0.6 x+4.7 y \leq 24.3, \\
& -x+3 y \leq 21, \\
& \min _{\left(y, n_{2}^{-}, v_{2}^{+}\right) \in Y^{\prime}} v_{2}^{-}+v_{2}^{+}, \\
& \text {subject to }-11.4 x+24.8 y+v_{2}^{-}-v_{2}^{+}=35, \\
& 0.6 x+2.2 y \leq 25.4, \\
& x+3 y \leq 7, \\
& 1.8 x+4.7 y \leq 30.2, \\
& x+3 y \leq 27,
\end{aligned}
$$

Using Branch-and-bound approach. " the colrent solution is (2.15366.0.0. $2.39243,0.0)$.

Step 4 (Comparison) Becanse $k=1$. go to Step 5 .

Step 5 (Splitting) By inserting a new node $\lambda_{1}=(0.2+1) / 2=0.6$. there are in total three nodes of $\lambda_{0}=0.2, \lambda_{1}=0.6$, and $\lambda_{2}=1$. Then, a total of 12 nonfuzzy objective functions for the leader and follower together with 12 nonfuzzy constraints for the leader and follower, respectively are generated.

Step 6 (Loop) $k=1+1=2$, go to Step 3, and the current solution of $(2.17093,0.0$. $2.41756,0,0)$ is obtained. As $|2.15366-2.17093|+|2.39243-2.41756|=0.04>\varepsilon=$ 0.01 , the algorithm keeps going until the solution of $(2.135,35,0.0 .2 .42797,0,0)$ is obtained. The computing results are listed in Table 1.

Step 7 (Output) As $|2.12393-2.13535|+|2.43436-2.12797|=0.0178<\varepsilon=0.02$, $\left(x^{*}, y^{*}\right)=(2.1354,2.4280)$ is the final solution of this $\mathrm{FGBL}$ decision problem. The

Table 1. Summary of the running solution.

\begin{tabular}{ccccccc}
\hline$k$ & $x$ & $y$ & $v_{1 \lambda}^{+}$ & $v_{1 \lambda}^{-}$ & $v_{2 \lambda}^{+}$ & $v_{2 \lambda}^{-}$ \\
\hline 1 & 2.15366 & 2.39243 & 0 & 0 & 0 & 0 \\
2 & 2.17093 & 2.41756 & 0 & 0 & 0 & 0 \\
3 & 2.12393 & 2.43436 & 0 & 0 & 0 & 0 \\
1 & 2.13535 & 2.42797 & 0 & 0 & 0 & 0 \\
\hline
\end{tabular}




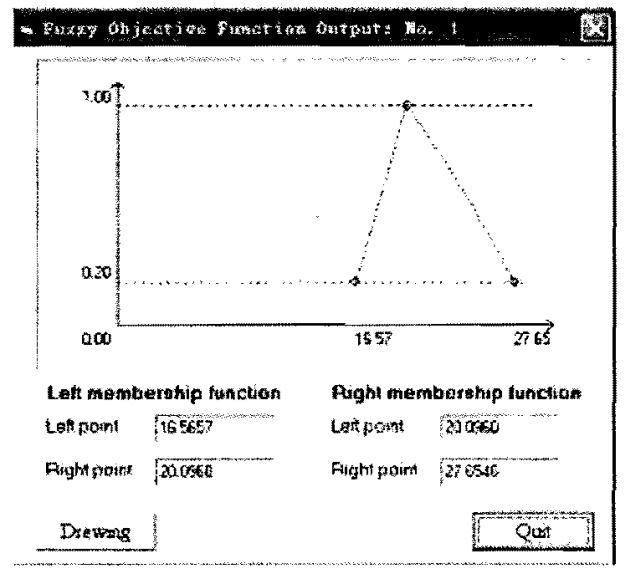

(a)

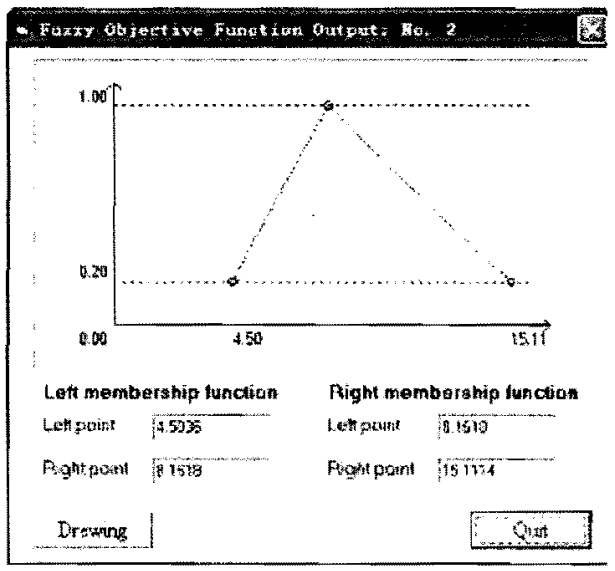

(b)

Fig. 2. Membership functions of $F^{N}\left(x^{*}, y^{*}\right)$ and $f\left(r^{*}, y^{*}\right)$.

objectives obtained for the lader and the follower under $\left(x^{*}, y^{*}\right)=(2.1354 .2 .4280)$ are

$$
\left\{\begin{array}{l}
F\left(x^{*}, y^{*}\right)=F(2.1354 .2 .4280)=2.1354 \tilde{c}_{1}+2.4280 \bar{d}_{1} \\
f\left(x^{*}, y^{*}\right)=F(2.1354,2.4280)=2.1354 \tilde{c}_{2}+2.4280 \tilde{d}_{2}
\end{array}\right.
$$

and their nembership functions are shown in Figs. $2(a)$ and $2(b)$.

The above example illustrated the detailed working process of the proposed algorithm.

\subsection{A case-based example on traffic management in a disaster area}

This section develops a case-based example on the traffe management in a disaster area by the FGBL model. When a disaster occurs, the blockage on roads and strects will cause severe problems for the nissions of evacuation. restoration, and rescue. It is necessary to balance the travel demand and servese supply in order to relieve traffic eongestion. This stuly addresses this problem from a two-level aspect to present the interactive decision process between the roadway control decision makers and the road user. Wo treat the commander of the Emergency-Response Center for the disaster-raided areas as the leater, whose objective $(F)$ is to allow traffic to go through the disaster area as much as possible under the condition of not exceeding the available roadway capacity. The road users, as the followers. will reasonably choose the shortest routes with regard to travel time, which are the objectives $\left(f_{1}\right.$ and $\left.f_{2}\right)$ for the followers. In the FGBL model. the decision maker for the Emergency-Response Center, the leader, controls the number of vehicles $(x)$ to enter the earthquake-raided area, while the road users, the followers, decide their 
specific route $\left(y_{1}\right.$ and $\left.y_{2}\right)$. The leader may have certain goal of traffic throughout $\left(\tilde{g}_{L}\right)$ for his or her objective, and the followers wish to meet the energency rescue needs $\left(\tilde{g}_{F 1}\right.$ and $\left.\tilde{g}_{F 2}\right)$ for their objective as well.

When modeling this problem, the main diffenity is fo set, 11 coefficients for the objectives and constraints of both the leader and the follower. We can only estimate these values according to our experience and previous data. Thus, by using fuzzy mumbers to describe these uncertain values, a FGBL model is established below:

$$
\begin{aligned}
& \max _{x \in \mathrm{X}} F\left(x, y_{1}, y_{2}\right)=\tilde{6} x+\tilde{3} y_{1}+\tilde{4} y_{2}, \\
& \text { subject to } \tilde{1} x+\tilde{3} y_{1}+\tilde{6} y_{2} \leq 2 \mathrm{1}, \\
& \min _{y_{1} \in Y_{3}} f\left(x, y_{1}\right)=\tilde{1} x+\tilde{3} y_{1}, \\
& \min _{y_{2} \in Y_{3}} f\left(x, y_{2}\right)=\tilde{3} x+\tilde{6} y_{2}, \\
& \text { subject to } \tilde{1} x+\tilde{3} y_{1}+\tilde{1} y_{2} \leq \tilde{1},
\end{aligned}
$$

where $x \in \mathbb{R} . y_{1} \cdot y_{2} \in \mathbb{R}_{:}$and $X=x \geq 0 . Y_{1}=y_{1} \geq 0, Y_{2}=y_{2} \geq 0$.

The membership functions of the coefficients of the objective functions and the construints of both the leader and the followers are as follows:

$$
\begin{aligned}
& \mu_{\tilde{0}}(x)=\left\{\begin{array} { l l } 
{ 0 } & { x < 5 , } \\
{ ( x ^ { 2 } - 2 5 ) / 1 1 } & { 5 \leq x < 8 , } \\
{ 1 } & { x = 6 , } \\
{ ( 0 4 - x ^ { 2 } ) / 2 8 } & { 6 < x < 8 , } \\
{ 0 } & { x > 8 , }
\end{array} \quad \mu _ { 3 } ( x ) \quad \left\{\begin{array}{ll}
0 & x<2, \\
\left(x^{2}-4\right) / 5 & 2 \leq x<3 \\
1 & x=3 \\
\left(25 \cdots x^{2}\right) / 16 & 3<x \leq 5 \\
0 & x>5
\end{array}\right.\right. \\
& \mu_{1}(x)=\left\{\begin{array}{ll}
0 & x<3, \\
\left(x^{2}-9\right) / 7 & 3 \leq x<1, \\
1 & x=4 . \\
\left(36-x^{2}\right) / 20 & 1<x \leq 6, \\
0 & x>6,
\end{array} \quad \mu_{1}(x)= \begin{cases}0 & x<0.5, \\
\left(x^{2}-0.25\right) / 0.75 & 0.5 \leq x<1, \\
1 & x=1, \\
\left(1-x^{2}\right) / 3 & 1<x \leq 2, \\
0 & x>2 .\end{cases} \right. \\
& \mu_{21}(x)= \begin{cases}0 & x<19 \\
\left(x^{2}-361\right) / 80 & 19 \leq x<21 \\
1 & x=21 \\
\left(625-x^{2}\right) / 181 & 21<x \leq 25 \\
0 & x>25\end{cases}
\end{aligned}
$$

The membership function of the fuzzy goal given to the learler is

$$
H_{x_{L 1}}(x)= \begin{cases}0 & x<15 \\ \left(x^{2}-225\right) / 175 & 15 \leq x<20 \\ 1 & x=20 \\ \left(900-x^{2}\right) / 500 & 20<x \leq 30 \\ 0 & x>30\end{cases}
$$


The membership functions of the fuzzy goals set for the followers are:

$$
\begin{aligned}
& \mu_{\tilde{g}_{F 1}}(x)= \begin{cases}0 & x<10 \\
\left(x^{2}-100\right) / 225 & 10 \leq x<15 \\
1 & x=15 \\
\left(400-x^{2}\right) / 175 & 15<x \leq 20 \\
0 & x>20\end{cases} \\
& \mu_{\tilde{g}_{F 2}}(x)= \begin{cases}0 & x<7 \\
\left(x^{2}-49\right) / 32 & 7 \leq x<9 \\
1 & x=9 \\
\left(121-x^{2}\right) / 40 & 9<x \leq 11 \\
0 & x>11\end{cases}
\end{aligned}
$$

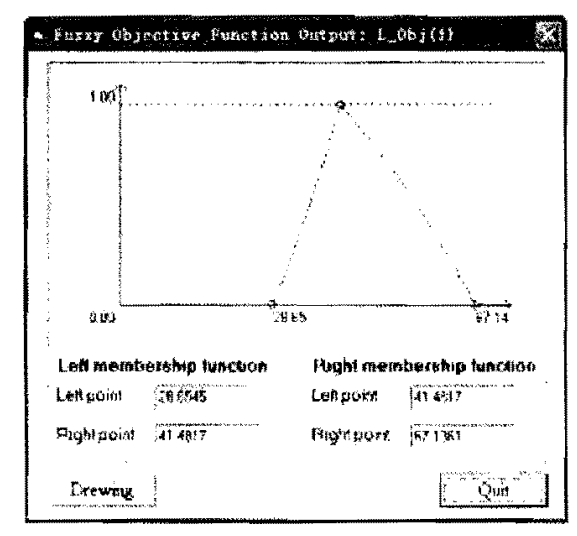

(a)

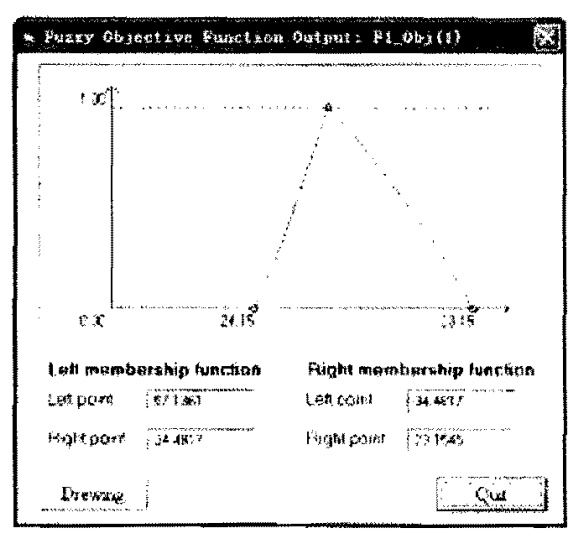

(b)

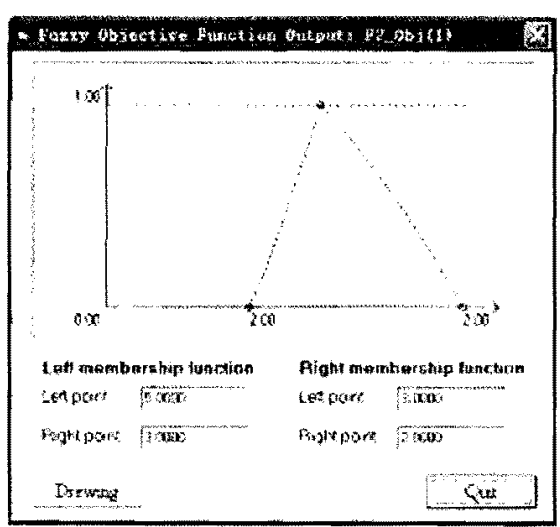

(c)

Fig. 3. Objectives for the leader and followers. 
Following all the steps of the proposed $\lambda$-cut approximate algorithm, the solution to this problem is: $\left(x^{*}, y_{1}^{*}, y_{2}^{*}\right)=(1.0,11.82,0.02)$. The objectives for the leader and followers under this solution are shown in Figs. 3(a) 3(c).

\section{Conclusion and Future Study}

Many organizational decision problems can be formulated by bilevel decision models. In a bilevel decision model. the leader and/or the follower may wish that their objectives attain some goals, which are different from simple optinization problems. This kind of bilevel decision problems are studied by goal programming in this paper. Meanwhile, we take into consideration of the sitnation where coefficients which formulate a bilevel decision model are not precisely known to us. Fuzzy set method is the applied to handle these coefficients.

This paper has proposed an approximate algorithm to solve FCBL decision problems, demonstraterl the software svstem, and presented two examples to further explain this algorithm. In the future. we will develop a method to handle the situation where the leader and the follower in a FGBL problem have multiple objectives, respectively.

\section{Acknowledgment}

The work presented in this paper was supported by Anstralian Research Comeil (ARC) under discovery grant DP0557154.

\section{References}

1. I. Amat and B. McCarl, A representation and oconomic interprestation of a two-level programming problem, J. Oper. Res. So. 32 (1981) $783 \cdots 792$

2. J. F. Bard, Prachenl Bitenet Optimization: Alyorithms and Applications (Kluwer Aca demic: Publishers, Boston, 1998).

3. W. Candler and R. Townsley, A lincar two-level programming prohlom, Comm. Oper. Res. 9 (1982) $59-76$

4. A. Charnes and W. W. Cooper. Management Models and Industrut Apptications of Linear Progmmming. Vol. I. (Wiley, 1961).

5. A. Charnes and W. W. Cooper, Coal programming and multiple objective optimizations, Eur. I. Oper. Res. 1 (1977) 39-54.

6. C. Feng and ( $\because$ Wen, Bi-level and multi-objective model to control traffic fow into the disaster area post earthquake, I. Eastern Asia Soc. Trunsport. Studies 6 (2005) $4253-4268$

7. Y. Gao, G. Zhang, J. Lu and S. Gao, A bilevel model for railway train set organizing optimization, in 2007 Int. Conf. Intelligent Syslems and Knowledge Engineering (ISKEQ007) (Atlantis Press, 2007): pp. 777 - 782.

8. Y. Gao, G. Zhang, J. Lu and X. Zeng, A $\lambda$-cut approximate approach to supporting fuzzy goal based bilevel decision making in risk management. in The First Int. Conf. Risk Analysis and Crisis Response. (Atlantis Press, 2007), pp. 132-137.

9. B. F. Hobbs, B. Metzler and I. S. Pang. Strategic gaming analvsis for electric power system: An mper approach, IEEE Trans. Powee System 15 (2000) 637645. 
10. C. Hu, C. Teng and S. Li, A fuazv goal programming approach to multi-objective optimization problem with priorities, Eur. J. Oper. Res. 176(3) (2007) 13191333.

11. J. P. Ignizio, Goal Programming and Extensions (Lexington Books, Massnchusetts, $1976)$.

12. J. P. Ignizio, Generalized goal programming: An overview. Comp. Oper. Res. 10 (1983) $277 \cdots 289$

13. Y. Lai, Hierarchical optimization: A satisfactory solution, Fuzzy Sets Syst. 77 (1996) 321335 .

14. S. M. Lee, Goal Programming for Decision Analysis (Auerbach Publishers, 1972).

15. S. Li, H. Wu and Y. Yang, Receding holizon fuzzy optimization under local information environment with a case study, hut. f. Infom. Technol. Decision Making 3(1) (2004) 109127

16. J. Lu. C. Shi, C. Zhang and T. Dillon, Nodel and extended Kuln Tucker approad for bilevel multi-follower decision making in a referential-mooperative situation, Int. J. Global Optimization 38(4) (2007) 597 .608.

17. J. Lu, $C$. Shi and $G$, Zhang, On bilevel multi-follower decision making: General framework and solutions. Inform. Set. 176(11) (2006) $1607 \cdots 1627$.

18. J. Lu. F. Wh and G. Zhang. On generalized fuzzy goal optimization for solving fuzzy multi-objective linear programming prohlens, J. Intell. Fizzy Syst. 18(1) (2007) 8397

19. \$. Pramanik and T. K. Roy. Fuzzy goal programming approach to nultilevel programming problems, Eur. J. Oper, Res. 176(2) (2007) 1151.1166.

20. O. M. Saad, An iterative goal programming approach for solving fuzzy multiobjective integer linear progranming problems, Appl. Math. Compul. 170(1) (2005) 216-225.

21. M. Sakawa, Fundamentals of fuzzy set theory, in Fuzzy Sets and Interactive MrultiObjective Optimizalion (Plenum Press, New York, 1993).

22. M. Sakawa, I. Nishizaki and Y. Uemura, Interactive fuzzy progremming for multilevel linear programming problems with fuzzy parameters, Fuzzy Sets Syst. 109 (2000) $3 \cdots 19$.

23. C. Shi, J, Lu and (6. Zhang. An extended Kuhn Tucker approach for linear bilevel programming. Appl. Math. Comp. $162(2005) 5163$.

24. H. S. Shih. Y. J. Lai and E. S. Lee. Fuzzy approach for multi-level programming problems, Comp. Oper. Res. 23 (1996) 7391.

25. H. Y. Stackelberg, Theory of the Market Economy (Oxford Dniversity Press. New York. 1952).

26. R. Stulz, Risk management and derivatives. Tech. Rep. (Thomson South-Western, Mason, Ohio. 2003).

27. M. Wang, H. Wang and C. Lin, Ranking fuzzy mumber hased on lexicographir screening procedure, Int. I. Inforn. Technol. Decison Making 4(4) (2005) 603-678

28. H. Yu, C. Dang and S. Wang, Game theoretical analysis of buy-it-now price auctions, Int. I. Inform. Technol. Decision Making 5(3) (2006) 557 - 581.

29. G. Zhang and J. Lu, Model and approach of fuzzy bilevel decision making for logistics planning problem. J. Enterprise fnform. Mngment 20(2) (2007) $178-197$.

30. C. Zhang. J. Lu and T. Dillon, An approximation Kuhn Tucker approach for fuzzy linear bilevel decision making problems, in Intel. Dects. Making. ods. I. Jain and G. Wren (Springer; 2007). 